\title{
Evaluation of high risk screening protocol for detection of overt hypothyroidism in pregnancy
}

\author{
Bharti Goel $^{1 *}$, Anju Singh², Poonam Goel ${ }^{1}$, Jasbinder Kaur ${ }^{3}$, Seema Singla ${ }^{3}$, Mandeep Singla ${ }^{4}$
}

\begin{abstract}
${ }^{1}$ Department of Obstetrics and Gynecology, Government Medical College and Hospital, Chandigarh, India ${ }^{2}$ Department of Obstetrics and Gynecology, Postgraduate Institute of Medical Education and Research, Chandigarh, India

${ }^{3}$ Department of Biochemistry, ${ }^{4}$ Department of Medicine, Government Medical College and Hospital, Chandigarh, India
\end{abstract}

Received: 21 August 2018

Accepted: 27 September 2018

*Correspondence:

Dr. Bharti Goel,

E-mail: bhartigoel14@gmail.com

Copyright: (C) the author(s), publisher and licensee Medip Academy. This is an open-access article distributed under the terms of the Creative Commons Attribution Non-Commercial License, which permits unrestricted non-commercial use, distribution, and reproduction in any medium, provided the original work is properly cited.

\section{ABSTRACT}

Background: Overt hypothyroidism is a known cause of feto-maternal morbidity. Many large-scale studies do not support the identification and treatment of sub-clinical hypothyroidism. Hence, we need a screening protocol that will identify all cases of overt hypothyroidism. The present study aimed to evaluate the high-risk screening protocol for detection of overt hypothyroidism during pregnancy.

Methods: Authors performed a prospective observational study for detection of thyroid dysfunction in 604 pregnant women in a tertiary care hospital setting. Detailed demographic, medical and obstetric history was noted and baseline serum thyrotropin (TSH) level and urinary iodine levels were checked. Reflex testing for thyroid peroxidase antibody was done in women diagnosed to have hypothyroidism. The enrolled women were then grouped as high risk if any of the high-risk criteria provided by ATA was positive.

Results: The study population was iodine sufficient with median urinary iodine (MUI) level of $255 \mu \mathrm{g} / \mathrm{l}$. Overall $32.2 \%$ women $(n=201)$ were found to be hypothyroid $(\mathrm{TSH}>2.5 \mathrm{mIU} / \mathrm{L}), 0.8 \%$ women $(\mathrm{n}=5)$ were hyperthyroid. Overt hypothyroidism was seen in $3.8 \%$ women $(n=23)$, all of whom were in the high-risk group as per the ATA guidelines. This co-relation was highly significant (likelihood ratio 24.94; P <0.001). Sensitivity of targeted screening for detection of overt hypothyroidism was $100 \%$. On the other hand, logistic regression analysis showed poor corelation of high-risk factors with sub-clinical hypothyroidism (TSH levels between $2.5 \mathrm{mIU} / \mathrm{L}-10.0 \mathrm{mIU} / \mathrm{L}$ ), likelihood ratio being $0.534 ; \mathrm{P}>0.05$.

Conclusions: High risk screening protocol is highly sensitive for detection of overt hypothyroidism and provides the best therapeutic payoff.

Keywords: High risk screening, Overt hypothyroidism, Pregnancy, Sub-clinical hypothyroidism, Targeted screening

\section{INTRODUCTION}

Overt hypothyroidism is a known cause of feto-maternal morbidity, though the association of subclinical hypothyroidism with adverse pregnancy outcome is controversial. ${ }^{1}$ Since prompt detection and treatment of overt disease is essential, the American Association of
Clinical Endocrinologists (AACE) and the American Thyroid Association (ATA) issued guidelines for high risk screening (targeted case finding approach) for thyroid disorders during pregnancy. ${ }^{2}$ On the other hand, the American College of Obstetricians and Gynecologists issued guidelines in 2015 (ACOG 2015), recommending against routine screening because some large scale 
studies had shown that identification and treatment of maternal subclinical hypothyroidism did not benefit fetal or maternal health. ${ }^{3-6}$ Hence, the ACOG recommends thyroid function assessment only in women with history of thyroid disease or symptoms of thyroid disease. ${ }^{3}$ In comparison, the ATA 2017 guidelines recommend that all newly pregnant women at high risk of thyroid disease should be identified by detailed history and clinical examination and investigated with a baseline serum thyrotropin (TSH) level which should be performed at the time of confirmation of pregnancy; along with reflex antithyroid peroxidase antibody (TPOAb) levels, if TSH is 2.5-10mU/L. ${ }^{1}$ Another school of thought recommends universal screening criticizing high risk screening because of its failure to identify a substantial number of pregnant women with thyroid dysfunction. ${ }^{7,8}$ However, none of these studies have focused on accuracy of highrisk screening protocol for detection of overt hypothyroidism which is the main cause of feto-maternal morbidity. With this background, the present study was conducted to evaluate the accuracy of this protocol for detection of overt hypothyroidism in pregnant women.

\section{METHODS}

The present study was a prospective observational study conducted in the outpatient clinic of Obstetrics and Gynecology Department in collaboration with the Department of Biochemistry, in a tertiary health care centre.

Based on the existing data from Indian sub-continent where the overall prevalence of hypothyroidism among pregnant women is about $10 \%$, authors calculated a sample size of 553 for a confidence level of $95 \%$ and precision of 0.025 . To account for errors in sample collection a total of 617 pregnant women were recruited in a consecutive manner.

\section{Inclusion criteria}

All pregnant women attending Gynecology clinic during period from March 2014 to September 2014 irrespective of past thyroid status, period of gestation or booking status were included in the study.

\section{Exclusion criteria}

Those who were not willing to give an informed consent were excluded.

The detailed demographic, medical and obstetric information were obtained to rule out all possible causes of thyroid dysfunction. History to ascertain pre-existing thyroid disease including symptoms due to hypo- or hyperthyroidism, thyroid surgery or radioactive iodine exposure, history to rule out other autoimmune disorders in self or family and history of chronic illnesses such as diabetes mellitus or chronic hypertension was sought. Obstetric information including number of previous pregnancies, history of infertility, early pregnancy loss, preterm birth and its cause, history of diabetes or preeclampsia in present or previous pregnancies, history of intrauterine growth restriction or fetal demise, and history of placental abruption was recorded. Detailed menstrual history was obtained. Clinical examination including assessment for Body Mass Index (BMI) and goitre was done, and the findings were recorded in a pre-designed format. The enrolled women were then grouped as high risk if any of the high-risk criteria provided by ATA 2017 were positive.

Blood samples for TSH and urine samples for spot urinary iodine excretion (UIE) were collected at the first visit. Women with TSH values more than $2.5 \mathrm{mIU} / \mathrm{L}$ in $1^{\text {st }}$ trimester and $3.0 \mathrm{mIU} / \mathrm{L}$ in $2^{\text {nd }}$ and $3^{\text {rd }}$ trimester but less than $10.0 \mathrm{mU} / \mathrm{L}$ were diagnosed with subclinical hypothyroidism (SCH) as per the ATA 2011 guidelines. ${ }^{9}$ Those with TSH levels > $10.0 \mathrm{mIU} / \mathrm{L}$ were diagnosed with overt hypothyroidism. Reflex testing for serum Thyroid Peroxidase Antibodies (TPOAbs) was performed in all subjects diagnosed with $\mathrm{SCH}$ or overt hypothyroidism. TSH and TPOAb levels were measured by chemiluminescence technique using commercially available kits by Advia Centaur XP analyzer system (Siemens Healthcare Diagnostics). The analytical sensitivities for TSH and TPO were $0.010 \mathrm{mIU} / \mathrm{L}$ and 28 $\mathrm{IU} / \mathrm{ml}$, respectively. Intra-assay coefficients of variation for TSH and TPOAb were $5.2 \%$ and $5.6 \%$ respectively. Laboratory reference range for TSH was $0.35-5.5 \mathrm{mIU} / \mathrm{L}$. A value of $>60 \mathrm{IU} / \mathrm{ml}$ was used to differentiate between TPOAbs positive or negative subjects as recommended by the manufacturer. Urinary iodine was measured by an assay based on Sandelt-Kolthoff reaction (wet digestion method). Pregnant women with urinary iodine excretion (UIE) less than $150 \mu \mathrm{g} / \mathrm{L}$ were considered as iodine deficient, those with levels between 150-249 $\mu \mathrm{g} / \mathrm{L}$ were considered to be iodine sufficient, between $250-499 \mu \mathrm{g} / \mathrm{L}$ were grouped as having more than adequate iodine while those with levels $>500 \mu \mathrm{g} / \mathrm{L}$ were considered as having excessive iodine as per WHO 2013 guidelines. ${ }^{10}$ The primary study parameter was assessment of the association of various high-risk factors with TSH levels and performance of high-risk approach for detection of overt thyroid dysfunction in pregnancy.

\section{Statistical analysis}

The normality of quantitative data was checked by measures of Kolmogorov-Smirnov tests of normality. Normally distributed continuous data was expressed as mean \pm standard deviation while the skewed data was expressed as median with inter-quartile range. Categorical data were represented in the form of number or percentage $(\%)$. Proportions were compared using Fisher's exact test or Chi-square test. To assess independent predictors for raised TSH, a Logistic Regression Analysis was applied. Spearman correlation coefficients were calculated to see relationship of TSH values with different variables. Analysis was conducted 
using IBM SPSS Statistics (version 22). All the statistical tests were two-sided and were performed at a significance level of $\alpha=0.05$.

\section{RESULTS}

At the end of study authors had complete data for clinical history, examination and investigations of 604 pregnant women. Spot urinary iodine excretion (UIE) levels were available for only 545 subjects due to loss of samples during handling and storage. The mean age and BMI were $26.21 \pm 3.78$ years and $23.04 \pm 2.39 \mathrm{~kg} / \mathrm{m}^{2}$ respectively. $12.4 \%$ women (75 of 604 ) were aged $>30$ years. Nine women $(1.5 \%)$ had a $\mathrm{BMI}>30 \mathrm{~kg} / \mathrm{m}^{2}$ while none had a BMI of $40 \mathrm{~kg} / \mathrm{m}^{2}$ or more. Trimester wise distribution of enrolled women was $66.6 \%$ women $(n=402), 28.3 \%$ women $(n=171)$ and $5.1 \%$ women $(n=31)$ in $1^{\text {st }}, 2^{\text {nd }}$ and $3^{\text {rd }}$ trimesters respectively. Using the predefined criteria, $24.8 \%$ women $(\mathrm{n}=150)$ in $1^{\text {st }}$ trimester and $8.4 \%$ women $(\mathrm{n}=51)$ in $2^{\text {nd }}$ and $3^{\text {rd }}$ trimester had TSH levels in the hypothyroid range. Amongst these, the prevalence of overt hypothyroidism was $3.8 \%(\mathrm{n}=23)$.

Eleven of these women (1.8\%) were diagnosed during the current pregnancy and remaining $12(2 \%)$ had been diagnosed prior to this pregnancy. Five women $(0.8 \%)$ gave history of hyperthyroidism. They were well controlled on treatment with thiazoles. The median urinary iodine (MUI) level was $255 \mu \mathrm{g} / 1$, which was more than adequate as per WHO standards. ${ }^{13}$ Reflex testing for TPOAb in hypothyroid women revealed $38.7 \%(n=77)$ positivity. TPOAb positivity was high in women with overt hypothyroidism as compared to those with $\mathrm{SCH}$ (87\% versus $32 \%) ; \mathrm{P}<0.05$ (Table 1$)$.

Table 1: TPO and TSH levels.

\begin{tabular}{lll} 
& TPO $\leq 60 \mathrm{IU} / \mathrm{ml}(\mathrm{n}=124)$ & $\mathrm{TPO}>60 \mathrm{IU} / \mathrm{ml}(\mathrm{n}=77)$ \\
$\mathrm{SCH}^{*}(\mathrm{TSH} 2.5 / 3.0-10.0 \mathrm{mIU} / \mathrm{L})$ & $121(68 \%)$ & $57(32 \%)$ \\
$\mathrm{OH}^{\dagger}(\mathrm{TSH}>10.0 \mathrm{mU} / \mathrm{L})$ & $3(13 \%)$ & $20(87 \%)$ \\
\hline
\end{tabular}

*: SCH: as defined by ATA 2011 guidelines; $\uparrow: \mathrm{OH}$ : Overt hypothyroidism

Table 2: Distribution of risk factors for thyroid disease.

\begin{tabular}{|llll|}
\hline Risk factor & $\begin{array}{l}\text { No. of women with risk } \\
\text { factor (Percentages") }\end{array}$ & $\begin{array}{l}\text { No. of women with } \\
\text { TSH }>2.5 \text { mU/L }\end{array}$ & $\begin{array}{l}\text { No. of women with } \\
\text { TPOAbs level > 60 IU/ml }\end{array}$ \\
\hline Obesity BMI $>30 \mathrm{~kg} / \mathrm{m}^{2}$ & $9(1.5 \%)$ & $5(55.5 \%)$ & $2(40 \%)$ \\
\hline Age $>30$ years & $75(12.4 \%)$ & $32(42.7 \%)$ & $10(42.7 \%)$ \\
\hline $\begin{array}{l}\text { History of thyroid dysfunction/ symptoms } \\
\text { suggestive of thyroid dysfunction }\end{array}$ & $14(2.4 \%)$ & $12(85.7 \%)$ & $9(75 \%)$ \\
\hline Presence of goitre & $32(5.3 \%)$ & $18(56 \%)$ & $9(50 \%)$ \\
\hline Family history of thyroid dysfunction & $37(6.2 \%)$ & $18(48.6 \%)$ & $9(50 \%)$ \\
\hline History of IDDM/ other autoimmune disorders & $5(0.8 \%)$ & $2(40 \%)$ & $1(50 \%)$ \\
\hline History of pregnancy loss & $187(31 \%)$ & $69(36.9 \%)$ & $29(42 \%)$ \\
\hline History of recurrent pregnancy loss $(\geq 3)$ & $14(2.4 \%)$ & $4(28.6 \%)$ & $2(50 \%)$ \\
\hline History of preterm birth $(<37$ weeks) & $13(2.2 \%)$ & $4(30.8 \%)$ & $3(75 \%)$ \\
\hline History of intrauterine fetal demise & $4(0.6 \%)$ & $1(25 \%)$ & $1(100 \%)$ \\
\hline History of pre-eclampsia & $24(4 \%))$ & 7 & 3 \\
\hline History of placental abruption & $3(0.5 \%)$ & 1 & 1 \\
\hline History of infertility & $40(6.6 \%)$ & 18 & 8 \\
\hline History of surgery/ radiation/antithyroid drugs & Nil & Nil & Nil \\
\hline History of multiple prior pregnancies $(>=2)$ & $299(49.5 \%)$ & 98 & 36 \\
\hline
\end{tabular}

There was no significant correlation between signs and symptoms of thyroid dysfunction and TSH levels. Only 5 of the 11 newly diagnosed cases gave positive history of one or more of symptoms such as weight gain, lethargy, fatigue, cold intolerance, constipation and depression. These symptoms were non-specific as $33 \%$ of euthyroid pregnant women also had one of these symptoms. Only one hypothyroid woman $(0.2 \%)$ gave history of hoarseness of voice; while $5.3 \%$ women $(n=32)$ were detected to have goitre, $56 \%$ of them (18 of 32) were found to have TSH $>2.5 \mathrm{mIU} / \mathrm{L}$. The rest were euthyroid.
About $6.0 \%$ women $(n=36)$ gave history of infertility. Amongst these $13.9 \%$ women (5 of 36 ) had history of ovulatory dysfunction and had conceived after ovulation induction. Obstetric evaluation revealed history of two or more prior pregnancies in $49.5 \%(n=299)$ and one or more early pregnancy loss in $31 \%$ women $(n=187)$. History of preterm birth, pre-eclampsia, placental abruption and intrauterine fetal demise in previous pregnancy were present in $2.2 \%, 4 \%, 0.5 \%, 1.2 \%$ and $0.6 \%(n=13, n=24, n=3, n=4)$ of the studied subjects respectively. Family history of thyroid dysfunction was 
present in $6.2 \%$ women $(n=37)$. None of the other highrisk factors were seen in present subjects (Table 2).

Overall $58.9 \%$ pregnant women (356 of 604) had one or more high risk factors indicating a need for assessment of serum TSH levels. Logistic regression analysis showed that none of these risk factors had significant co-relation with TSH levels $>2.5 \mathrm{mIU} / \mathrm{L}$. Likelihood ratio (LR) was 0.534; P>0.05 (Table 3).

On the other hand, authors found significant association of high-risk factors with overt hypothyroidism. None of the women in the low risk group $(n=248)$ had overt hypothyroidism, while the likelihood ratio for TSH level $>10.0 \mathrm{mU} / \mathrm{L}$ in presence of one or more risk factors was 24.94; $\mathrm{P}<0.001$ (Table 4).

Further the sensitivity, specificity, negative predictive value (NPV), positive predictive value (PPV) and accuracy of targeted screening for detection of overt hypothyroidism was found to be $100 \%, 42.7 \%, 6.5 \%$, $100 \%$ and $44.9 \%$ respectively (Table 5). NPV of $100 \%$ implies that high risk screening identified all cases of overt hypothyroidism in present study.

Table 3. The odds ratio and $\mathbf{9 5 \%}$ CI of various risk factors for prediction of sub-clinical hypothyroidism.

\begin{tabular}{|l|lll|}
\hline Risk factors & Exp $(\mathrm{B})$ & $95 \%$ CI Exp (B) & P value \\
\hline History of infertility & 1.442 & $0.750-2.774$ & 0.272 \\
\hline Family history of thyroid disease & 1.600 & $0.816-3.140$ & 0.171 \\
\hline Multiple prior pregnancies & 0.703 & $0.432-1.145$ & 0.157 \\
\hline Previous pregnancy loss & 1.591 & $0.961-2.634$ & 0.071 \\
\hline Type 1 diabetes mellitus & 0.720 & $0.285-1.819$ & 0.487 \\
\hline History of preterm delivery & 1.032 & $0.325-3.277$ & 0.957 \\
\hline History of pre-eclampsia & 1.545 & $0.875-2.695$ & 0.315 \\
\hline Age >30 years & 1.237 & $0.734-2.083$ & 0.424 \\
\hline Presence of goitre & 1.505 & $0.798-3.785$ & 0.154 \\
\hline
\end{tabular}

Table 4: Association between overt hypothyroidism and the presence of a high-risk factor.

\begin{tabular}{|llllll} 
& Value & df & Asymp. Sig. (2-sided) & Exact Sig. (2-sided) & Exact Sig. (1-sided) \\
\hline Pearson chi-square & 16.657 (b) & 1 & 0.000 & & \\
\hline Continuity correction (a) & 14.940 & 1 & 0.000 & & 0.000 \\
\hline Likelihood ratio & 24.949 & 1 & 0.000 & 0.000 & \\
\hline Fisher's exact test & & & & & \\
\hline Linear-by-linear association & 16.629 & 1 & 0.000 &
\end{tabular}

Table 5: Association of TSH levels with risk factors for thyroid disease.

\begin{tabular}{|lllll} 
& Euthyroid $(\mathrm{n}=398)$ & Subclinical $(\mathrm{n}=178)$ & Overt $(\mathrm{n}=23)$ & Hyperthyroid $(\mathrm{n}=5)$ \\
\hline Risk $+(\mathrm{n}=356) *$ & $216(60.7 \%)$ & $114(32 \%)$ & $23(6.5 \%)$ & $3(0.8 \%)$ \\
Risk $-(\mathrm{n}=248)^{\dagger}$ & $182(73.4 \%)$ & $64(25.8 \%)$ & $0(0 \%)$ & $2(0.8 \%)$ \\
\hline
\end{tabular}

$*$ Risk factor present; $\uparrow$ Risk factor absent

On the other hand, the sensitivity, specificity, NPV, PPV and accuracy of targeted screening for detection of $\mathrm{SCH}$ was found to be $64 \%, 43.2 \%, 32 \%, 74.2 \%$ and $49.3 \%$ respectively (Table 5). These results reflect upon the weak association of various high-risk factors for $\mathrm{SCH}$.

\section{DISCUSSION}

Present study showed a $100 \%$ NPV and $100 \%$ sensitivity of high-risk screening protocol (targeted case finding strategy) for identification of overt hypothyroidism. Similar to present study, Yang et al reported $100 \%$ sensitivity of targeted screening for detection of overt hypothyroidism in 1 st trimester and concluded that targeted screening appears to work well in pregnant women with overt hypothyroidism. ${ }^{11}$ Similarly, Nazarpour et al reported sensitivity of $88.2 \%$ for overt hypothyroidism in their study. ${ }^{12}$

On the other hand, many other researchers have reported poor performance of high-risk screening approach for detection of thyroid dysfunction.

Chang et al reported that $80 \%$ of women with hypothyroidism could not be diagnosed using the highrisk case finding approach. ${ }^{7}$ Wang et al reported that this approach failed to diagnose $81.6 \%$ of pregnant women with hypothyroidism and $80.4 \%$ of pregnant women with 
hyperthyroidism. ${ }^{8}$ Ohashi et al also showed that high-risk case finding identified only $10 \%$ of women with thyroid dysfunction while $90 \%$ of women with thyroid dysfunction were not identified. ${ }^{13}$ Ahmed et al also reported that $34.5 \%$ of pregnant women with hypothyroidism were overlooked by high risk screening approach. ${ }^{14}$ However, there are two main points to be noted when analysing this literature. Firstly, these studies have not focused on the accuracy of targeted screening for diagnosing overt hypothyroidism. Most of these studies talk about cases with $\mathrm{SCH}$ that were missed on high risk screening. Secondly, these studies have not included the additional high-risk factors of age $>30$ years and history of multiple prior pregnancies $(\geq 2)$ which has been recommended by ATA 2017 guidelines. Addition of both these factors to the case-finding screening strategy substantially improved its efficiency in present study due to a larger number of women screened. This observation was also made by Potlukova et al in their study. ${ }^{15}$

The poor accuracy of targeted screening for detection of $\mathrm{SCH}(49.3 \%)$ can be explained by poor association of $\mathrm{SCH}$ with high risk factors; logistic regression analysis in present study showed LR of $0.534 ; \mathrm{P}>0.05$. The odds ratio for $\mathrm{SCH}$ (95\% confidence interval), in presence of high risk factors such as history of infertility, family history of thyroid disease, multiple prior pregnancies $(\geq 2)$, previous pregnancy loss, type 1 diabetes mellitus, preterm delivery, pre-eclampsia, age >30 years and presence of goitre were $1.442(0.750-2.774), 1.600$ (0.816-3.140), 0.703 (0.432-1.145), 1.591 (0.961-2.634), 60.720 (0.285-1.819), 1.032 (0.325-3.277), 1.545 (0.875$2.965), 1.237(0.734-2.083)$ and $1.505(0.798-3.785)$ respectively. None of these ratios were significant; $\mathrm{P}$ $>0.05$ (Table 3 ). These findings are supported by a recent Cochrane review which reported that despite the increase in the number of women diagnosed with hypothyroidism by screening for thyroid dysfunction in pregnancy, clearly evident improvement in maternal and fetal outcome in terms of pre-eclampsia, preterm birth, developmental delay and intellectual impairment in children at 3 years of age was lacking. ${ }^{16}$

The ACOG recommends against the routine high risk screening for thyroid dysfunction and recommends that TSH levels be tested only in women with personal or family history of thyroid disease or in those who have symptoms of thyroid disease. ${ }^{3}$ However, authors found that clinical diagnosis of overt hypothyroidism in pregnancy is not accurate, as authors could identify only 5 out of $11(45 \%)$ patients with overt hypothyroidism. This is because the symptoms of thyroid dysfunction are nonspecific and often overlap with pregnancy. Symptoms of hyperthyroidism such as nervousness, insomnia, palpitations, and hypertension, tachycardia, frequent stools, heat intolerance, and excessive sweating; and those of overt hypothyroidism such as fatigue, constipation, cold intolerance, muscle cramps and weight gain may be present during pregnancy, irrespective of thyroid dysfunction. Also, goitre may or may not be present with thyroid dysfunction. In fact, a physiological increase in size of thyroid gland can occur in pregnancy. ${ }^{1}$ This was also seen in present study where authors found that only $56 \%$ women $(n=18)$ with goitre were hypothyroid, while the rest $44 \%(n=14)$ were euthyroid. Prevalence rate of overt hypothyroidism in present study was $3.8 \%$. Similarly, Ajmani et al and Sahu et al reported a prevalence of $3 \%$ and $4.58 \%$ in their studies. ${ }^{18,19}$ Hence screening for overt hypothyroidism is important in our country. On the other hand, there is no definite evidence in literature to support the need for detection and treatment of SCH. Targeted high-risk case finding strategy is useful because it helps in efficient diagnosis of overt hypothyroidism and reduces the burden which will be imposed by universal screening protocol on the existing health care system. This rationale has also been supported by the guidelines issued by the Indian Ministry of Health and Family Welfare which supports the protocol of screening high risk pregnant women for thyroid dysfunction. ${ }^{20}$

Merits of the study: Present study has focused on the identification of overt hypothyroidism through the highrisk screening protocol by applying the factors recommended by ATA in 2017, hence improving the accuracy of this approach. Estimation of the median urinary iodine concentration in present study population, helped to rule out iodine insufficiency as a confounding factor for comparison of universal screening with high risk screening strategy.

Limitation and drawback of the study: Because of the low prevalence of hyperthyroidism, authors could not identify any new cases in present study population. Hence no comment was possible on the role of high-risk screening in detection of hyperthyroidism.

Future research direction: Since there is a positive correlation between autoimmune thyroid disease and increasing levels of TSH, indicating some thyroid dysfunction, role of targeted screening in identifying and treating these asymptomatic women who may be at high risk of adverse pregnancy outcomes and subsequent postpartum thyroiditis needs to be researched further.

\section{CONCLUSION}

The current recommendation of high-risk screening for thyroid dysfunction during pregnancy accurately identifies overt hypothyroidism, the most significant of thyroid dysfunctions, that needs definite treatment. Since the ultimate measure of a screening program is the probability that it will generate useful information that has some therapeutic pay-off, a high risk screening protocol built in empirically into good clinical practice, which is sensitive enough to identify all cases of overt hypothyroidism, has enormous advantage over a formal universal screening protocol and seems the most appropriate strategy at present. 


\section{ACKNOWLEDGMENTS}

Authors would like to acknowledge the support provided by the Professor and Head of the Department of Obstetrics and Gynaecology, Dr. Anju Huria. Authors would also like to acknowledge the efforts put in by Ms. Kusum Chopra and Ms. Deepti Dua who helped in compilation and statistical analysis of the data. Authors thank the patients because without their cooperation the study would not have been possible.

Funding: This research project was funded by a grant from the Department of Science and Technology, Chandigarh, India

Conflict of interest: None declared

Ethical approval: The study was approved by the Institutional Research and Ethics Committee

\section{REFERENCES}

1. Alexander EK, Pearce EN, Brent GA, Brown RS, Chen Herbert, Dosiou C, et al. Guidelines of the American Thyroid Association for the diagnosis and management of thyroid disease during pregnancy and the postpartum. Thyroid. 2017;27(3):315-89.

2. Garber JR, Cobin RH, Gharib H, Hennessey JV, Klein I, Mechanick JI, et al. Clinical practice guidelines for hypothyroidism in adults: cosponsored by the American Association of Clinical Endocrinologists and the American Thyroid Association. Endocr Pract. 2012;18(6):988-1028.

3. Thyroid Disease in Pregnancy: Practice Bulletin No. 148. Obstet Gynecol. 2015;125(4):996-1005.

4. Tudela CM, Casey BM, McIntire DD, Cunningham FG. Relationship of subclinical thyroid disease to the incidence of gestational diabetes. Obstet Gynecol. 2012;119:983-8.

5. Wilson KL, Casey BM, McIntire DD, Halvorson LM, Cunningham FG. Subclinical thyroid disease and the incidence of hypertension in pregnancy. Obstet Gynecol. 2012;119:315-20.

6. Lazarus JH, Bestwick JP, Channon S, Paradice R, Maina A, Rees R, et al. Antenatal thyroid screening and childhood cognitive function. $N$ Engl $J$ Med. 2012;366:493-501.

7. Chang DL, Leung AM, Braverman LE, Pearce EN. Thyroid testing during pregnancy at an academic Boston Area Medical Center. J Clin Endocrinol Metab. 2011;96(9): E1452-6.

8. Wang W, Teng W, Shan Z, Wang S, Li J, Zhu L, et al. The prevalence of thyroid disorders during early pregnancy in China: the benefits of universal screening in the first trimester of pregnancy. Eur J Endocrinol. 2011;164(2):263-8.

9. Stagnaro-Green A, Abalovich M, Alexander E, Azizi F, Mestman J, Negro R, et al. Guidelines of the American Thyroid Association for the diagnosis and management of thyroid disease during pregnancy and postpartum. Thyroid. 2011;21(10):1081-125.
10. WHO. Urinary iodine concentrations for determining iodine status deficiency in populations. Vitamin and Mineral Nutrition Information System. Geneva: World Health Organization; 2013: WHO/NMH/NHD/EPG/13.1. Available at: http://www.who.int/nutrition/vmnis/indicators/urinaryio dine.

11. Yang H, Shao M, Chen L, Chen Q, Yu L, Cai L, Lin Z, et al. Screening Strategies for thyroid disorders in the first and second trimester of pregnancy in China. PLoS One. 2014;9(6):e99611

12. Nazarpour S, Tehrani FR, Simbar M, Tohidi M, AlaviMajd H, Azizi F. Comparison of universal screening with targeted high-risk case finding for diagnosis of thyroid disorders. Eur $\mathrm{J}$ Endocrinol. 2016;174(1):77-83.

13. Ohashi M, Furukawa S, Michikata K, Kai K, Sameshima H, Ikenoue T. Risk-based screening for thyroid dysfunction during pregnancy. J Preg. 2013;2013.

14. Ahmed IZ, Eid YM, El Orabi H, Ibrahim HR. Comparison of universal and targeted screening for thyroid dysfunction in pregnant Egyptian women. Eur J Endocrinol. 2014;171:285-91.

15. Potlukova E, Potluka O, Jiskra J, Limanova Z, Telicka $\mathrm{Z}$, Bartakova J, et al. Is age a risk factor for hypothyroidism in pregnancy? An analysis of 5223 pregnant women. J Clin Endocrinol Metab. 2012;97:1945-52

16. Spencer L, Bubner T, Bain E, Middleton P. Screening and subsequent management for thyroid dysfunction pre-pregnancy and during pregnancy for improving maternal and infant health. Cochrane Database Syst Rev. 2015;9:CD011263.

17. Cignini P, Cafà EV, Giorlandino C, Capriglione S, Spata A, Dugo N. Thyroid physiology and common diseases in pregnancy: review of literature. J Prenat Med. 2012;6(4):64-71.

18. Ajmani SN, Aggarwal D, Bhatia P, Sharma M, Sarabhai V, Paul M. Prevalence of Overt and subclinical thyroid dysfunction among pregnant women and its effect on maternal and fetal outcome. J Obstet Gynaecol India. 2014;64(2):105-10.

19. Sahu MT, Das V, Mittal S, Agarwal A, Sahu M. Overt and subclinical thyroid dysfunction among Indian pregnant women and its effect on maternal and fetal outcome. Arch Gynecol Obstet. 2010;281(2):215-20.

20. National Guidelines for Screening of Hypothyroidism during Pregnancy. Maternal Health Division Ministry of Health and Family Welfare Government of India December 2014. Available at: http://nhsrcindia.org/sites/default/files/Guidelines\%20fo r\%20Maternal\%20Near\%20Miss.pdf.

Cite this article as: Goel B, Singh A, Goel P, Kaur J, Singla S, Singla M. Evaluation of high risk screening protocol for detection of overt hypothyroidism in pregnancy. Int $\mathbf{J}$ Reprod Contracept Obstet Gynecol 2018;7:4581-6. 